\section{Control and precision in complete arch restoration}

\author{
B. Mizrahi ${ }^{1}$
}

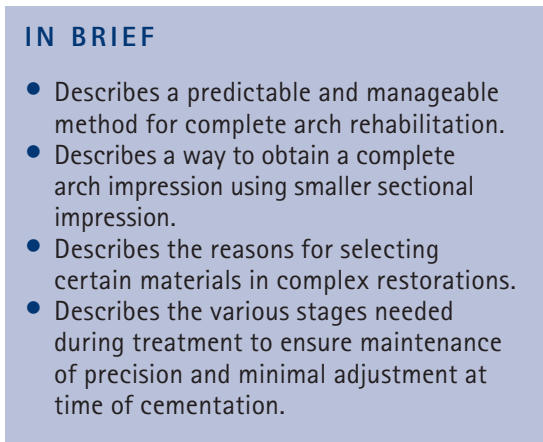

There are certain advantages to restoring a complete arch simultaneously, however, attempting to do this requires that the dentist maintains control during all stages of treatment. Maintaining control alleviates the preassure of time, which in turn allows for precision to be obtained at all clinical and laboratory stages. Two potential areas where control may be diminished or lost are: i) the construction and utilisation of temporary restorations that are biomechanically sound, durable, aesthetic and make a significant contribution to the final result; and ii) obtaining a master cast with accurate dies of all the tooth preparations. This article discusses a logical and practical approach to complete arch restorations and describe the advantages of moving from initial temporary restorations through laboratory processed, provisional restorations and then on to the final restorations. A practical approach for obtaining a single cast with acurate dies of all the tooth preparations is also discussed.

\section{Control and precision in complete arch restoration}

When faced with a patient who has a failing dentition where complete arch restoration is indicated, the dentist needs to decide whether to place all the final restorations simultaneously or treat the case in smaller sections.

Advantages to completing a complete arch of restorations simultaneously include:

- Freedom to reconfigure the occlusal relationship if needed

- Improved laboratory control/design of the occlusal relationship and aesthetic appearance.

Due to the nature of materials and equipment used, it is inevitable that minor discrepancies will exist between the restorations in the dental laboratory and the mouth. In order to keep such

\footnotetext{
Specialist in Prosthodontics and Restorative Dentistry, Fellow, American College of Prosthodontists, Clinical Lecturer, UCL Eastman Dental Institute

Correspondence to: Dr Basil Mizrahi, 39 Harley Street,

London, W1G 80H

Email:info@basilmizrahi.co.uk
}

\section{Refereed Paper}

Accepted 24 October 2007

DOI: $10.1038 /$ bdj.2008.3

${ }^{\oplus}$ British Dental Journal 2008; 204: 69-75 discrepancies to a minimum it is essential for the clinical and laboratory stages to be carried out to the highest possible level of precision and accuracy. Treatment needs to be carried out in stages, ensuring the success of each stage before proceeding on to the next stage. At each stage, it is necessary to carry out intra-oral and extra-oral checks for discrepancies and if necessary, make adjustments to correct these. If these discrepancies are not kept to an absolute minimum and corrected along the way, they will magnify as treatment proceeds creating large errors at the end of treatment. Such checks include:

- Verification of interocclusal records as treatment proceeds

- Aesthetic try in of ceramic restorations in pre-finished state

- Internal fit checking and adjusting of restorations before cementation.

In all aspects of dentistry, time is an essential factor to allow for the achievement of clinical and technical precision. When restoring a complete arch, this time requirement is magnified proportionally and treatment is usually carried out in stages over an extended period of time. During this time, it is important for the dentist to maintain control of the case and ensure that at all stages, the patient's dentition is biologically stable, functionally comfortable and aesthetically pleasing. Initial temporary restorations followed by laboratory processed provisional restorations are essential stages in allowing the dentist to maintain control and take the amount of time required to achieve clinical and laboratory precision.

To restore a complete arch simultaneously, it is necessary to obtain a complete arch impression or a master model containing accurate and precise reproductions of all the tooth preparations. When assessed carefully under appropriate magnification (10x), with an understanding and knowledge of impression errors, it becomes apparent that achieving an accurate complete arch impression on a predictable basis is all but impossible. Often, when it has not been possible to successfully impress all the tooth preparations, the main impression is supplemented with additional sectional impressions of the inadequately impressed teeth. The technician is then left with the option of either uniting all the dies on one master cast, or working on two different models: one for the margins, and one for the contact points and occlusion. ${ }^{1,2}$ It has been shown, however, 
that it is not possible to obtain identical dies from two different impressions (including reversible hydrocolloid), and as such, the accuracy of such techniques can be questioned. ${ }^{3}$

The following case outlines the stages in treatment of a complete a complex complete arch rehabilitation. It illustrates the steps necessary in progressing from initial temporary restorations to definitive provisional restorations and then finally on to the final restorations. A technique and the steps utilised therein, to obtain a cast with accurate impressions of all preparations, is also described.

\section{CASE REPORT}

The patient was a 60-year-old female with no medical problems. She had a history of continuous dental treatment over her lifetime with various dentists. Her main desire was to improve the health and appearance of her teeth and the overall comfort of her mouth.

On examination it was evident that most of the upper teeth were heavily restored with composites, amalgams and crowns. An upper left premolar was missing and this had allowed the upper arch to become asymmetrical with a midline shift toward the left side (Figs 1-3).

Occlusal examination revealed a discrepancy between centric relation and centric occlusion and multiple excursive interferences. There was no TMJ or muscle discomfort. The lower arch was relatively stable with minimal restorations.

Treatment was carried out in the following stages:

1. Clinical records for treatment plan-

ning and initial basic diagnostic wax-up

2. Establishment of biologically sound foundation restorations and placement of initial temporary restorations

3. Laboratory processed, provisional restorations

4. Final impressions

5. Fitting and cementation of definitive restorations.

\section{Stage 1 - Clinical records and initial wax-up}

Despite the fact that complex treatment was being carried out, with cor- rect knowledge and understanding, it is possible to use simplified instrumentation. ${ }^{4,5}$ As such, the study casts were mounted using a facebow and a centric relation record on a semi adjustable articulator. Following careful assessment of all the records and a discussion with the patient, the treatment plan was formulated and called for ceramo-metal crowns on all the upper teeth except teeth 17 and 27 which were to be restored with gold onlays.

Ceramo-metal crowns rather than allceramic crowns were used on the anterior teeth because they allow for more conservative, metal chamfer margins to be placed in non aesthetic areas. The potential aesthetic advantage of allceramic crowns was negated by the fact that there was adequate labial space (2 $\mathrm{mm}$ ) to develop good aesthetics and colour matching was not an issue as all the teeth were being crowned.

A duplicate set of study casts was fabricated and cross mounted to the first set of casts. An initial wax-up was made on the duplicate set of casts at a slightly increased (2 $\mathrm{mm}$ anteriorly) vertical dimension of occlusion (VDO). The decision to increase the VDO was empirical and based on the aesthetic requirement of increased anterior tooth length and the restorative requirement of sufficient supragingival tooth structure to establish adequate resistance form. ${ }^{6}$

At this early stage of treatment it is not necessary or desirable to fabricate a definitive wax-up and laboratory processed, provisional restorations. All that is required is a basic wax up on which to base the initial chairside temporary restorations.

\section{Stage 2 - Initial chairside temporary restorations}

Over a series of appointments, the old restorations were removed and new foundation restorations were placed where necessary. Chairside fabricated temporary crowns and onlays made from matrices of the initial wax up were then placed at the new VDO (Fig. 4).

The primary objective of this stage is to prepare the teeth and place immediate temporary restorations in readiness for placement of laboratory processed, provisional restorations at a later stage.

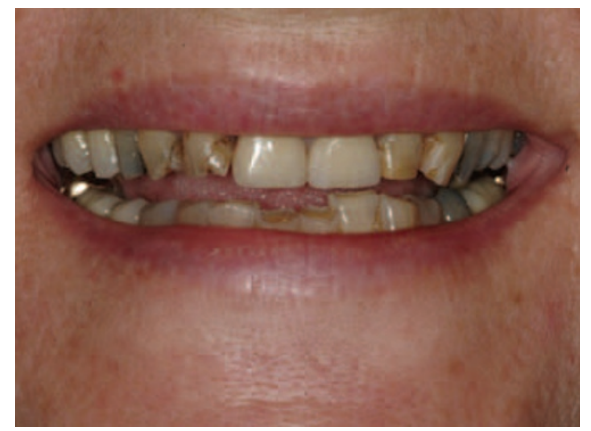

Fig. 1 Smile. Pre-op

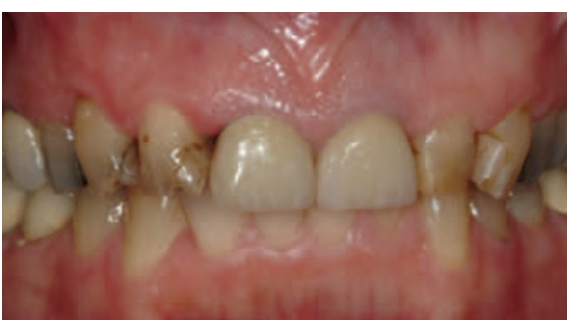

Fig. 2 Anterior view. Pre-op

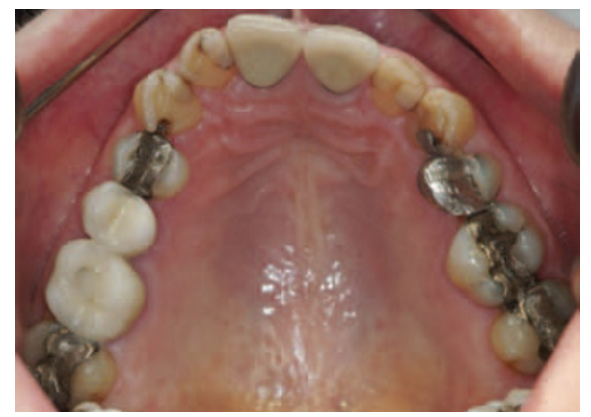

Fig. 3 Maxillary arch. Pre-op. Note missing left premolar and asymmetrical arch form

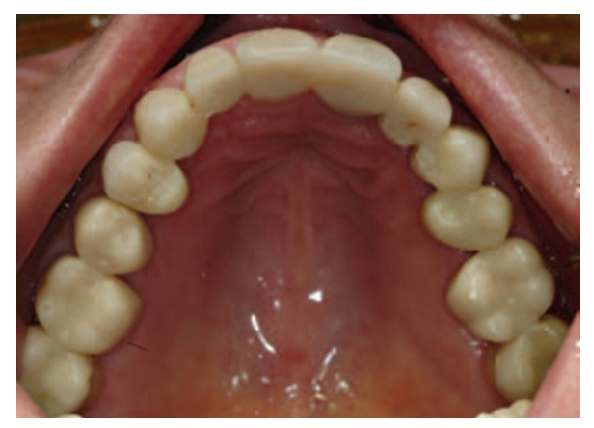

Fig. 4 Initial chairside temporary restorations in place

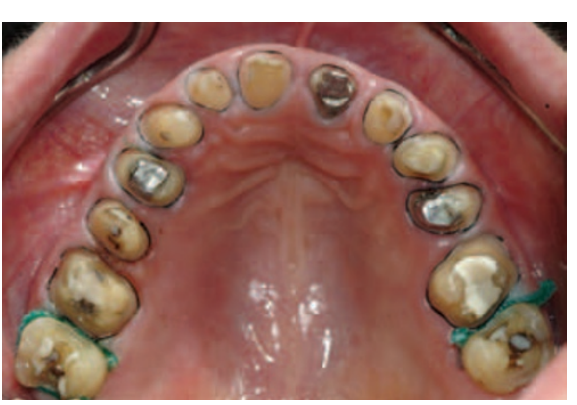

Fig. 5 Completed tooth preparations and core build-ups ready for impression for definitive provisional restorations and gold onlays on teeth 17 and 27 


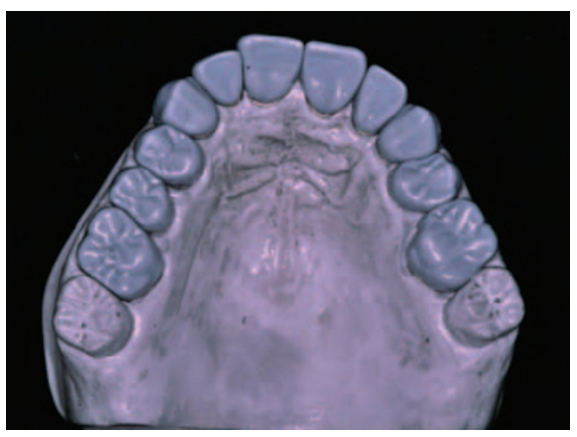

Fig. 6 Definitive wax up

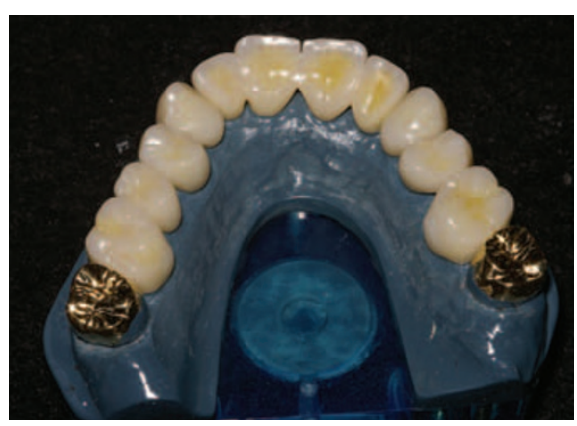

Fig. 7 Laboratory processed, provisional restorations and gold onlays

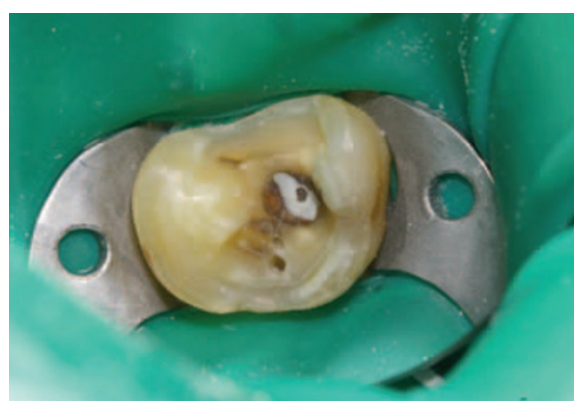

Fig. 8 Tooth 27 ready for cementation of gold onlay under rubber dam with Zinc Phosphate cement. Note retentive pin hole for Platinum-Irridium pin incorporated in gold casting

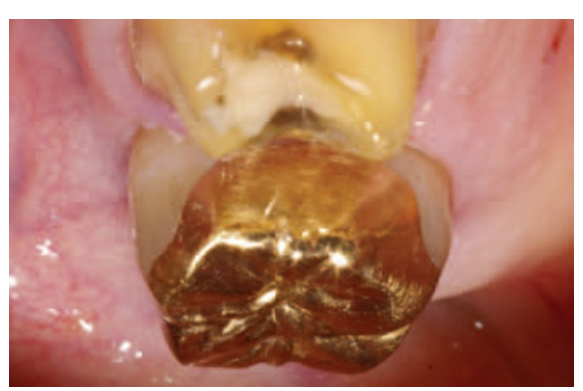

Fig. 9 Cemented gold restoration on tooth 27. Note smooth transition between gold and tooth due to burnishing of margins

This can be carried out within the dentist's comfort zone over as many appointments as necessary using a variety of techniques. ${ }^{7}$ If the VDO is being altered, as in this case, teeth that have not yet been prepared can be temporarily built up directly in the mouth with composite
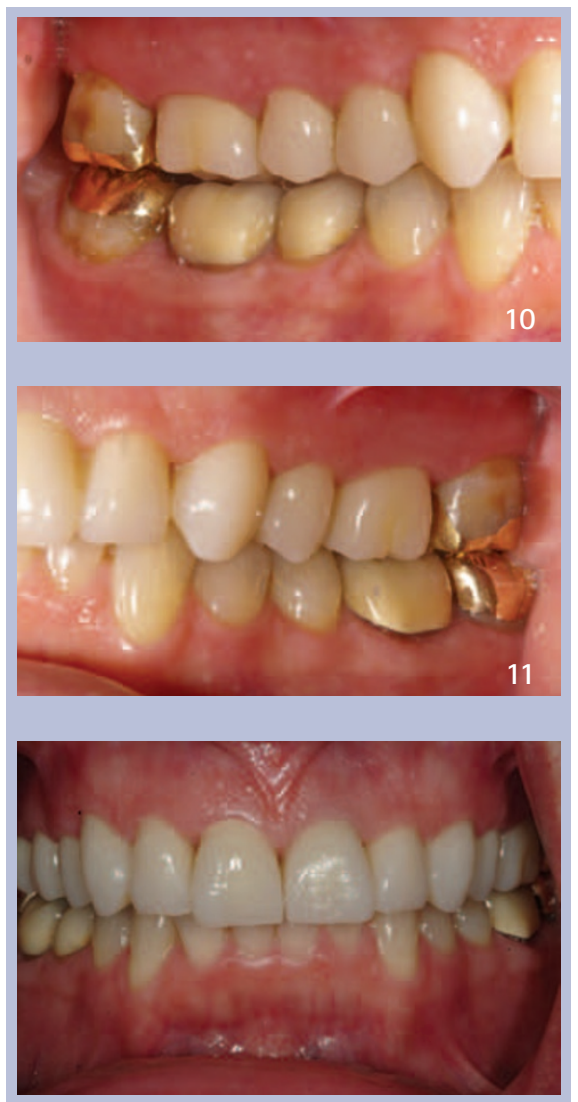

Figs 10-12 Definitive gold and laboratory processed, provisional restorations in place

resin until such time as they are temporised at the new VDO.

Methylmethacrylate, powder-liquid, acylic systems rather than bis-acryl based, automix paste-paste, systems are the optimal material to use for the temporary restorations in complex cases. Because of its lower modulus of elasticity, acrylic resin can be removed and replaced more easily. Methylmethacrylate also has a high transverse repair strength which allows for remargination and shape modification. ${ }^{8}$ It also has more versatile mixing/application options making it more amenable to modification.

During this initial temporisation stage, the following is carried out over multiple appointments:

- Aesthetic and functional modifications are made to the temporaries. The starting point for determining correct tooth position and appearance is the incisal edge position which is based upon aesthetics, phonetics and function. These three aspects can only be assessed during function in the mouth and as such, it is not possible to precisely determine incisal edge position from the wax up alone. The definitive incisal edge position can only be determined in the mouth by means of temporary crowns which are modified as needed over a period of time ${ }^{9}$

- Adjunctive treatment such as endodontic and or periodontic treatment is completed

- All core restorations are completed

- Tooth preparations are finalised

- The temporary restorations are relined as the tooth preparations are modified, refined and finalised over time.

If the initial temporary stage is bypassed, the laboratory processed, provisional restorations will not be precisely fitting, as the teeth will not yet have been prepared. ${ }^{10}$ Any occlusal and marginal precision obtained in the laboratory will be lost when the provisionals are transferred to the mouth and relined. In addition, while adjunctive treatment is carried out and tooth preparation finalised, continual remargination of the provisionals will lead to occlusal discrepancies and soft tissue inflammation.

By the end of this stage, all prepared teeth should be in temporary restorations; core restorations should be biomechanically sound and adjunctive treatment and tooth preparation should be completed (Fig. 5).

\section{Stage 3 - laboratory processed provisional restorations}

At this time, once soft tissue health is evident, a complete arch impression is made for fabrication of the laboratory processed, provisional restorations in the laboratory. This impression is made in a similar way to that for the final restorations. All attempts should be made to capture as much of the preparation margins as possible and if necessary small amounts of retraction cord should be used. Minor imperfections are however acceptable as the provisional restorations can be intra-orally remarginated in isolated areas, if necessary.

It is at this stage that the true skills and ability of the dental technician are bought into play. Once the technician has an impression of the tooth preparations s/he can then carry out a definitive wax-up based on information gathered from the initial temporary restorations. 
This definitive wax up should take into account aesthetic, occlusal and functional requirements and represent the precise outcome desired from the final restorations (Fig. 6).$^{11}$ The definitive provisional restorations are then fabricated from the definitive wax up, using heat polymerised methylmethacrylate acrylic resin. It was decided to fabricate the definitive gold onlays (Jensen JRVT Gold 77\%, Silver 13\%, Copper 8.5\%, Palladium 1\%) on teeth 17 and 27 at the same time as making the provisional restorations (Figs 8-9). The reason for this was to maintain the VDO during the extended period of time that the provisional restorations were to be in place and to reduce the time that partial coverage temporary restorations were in place.

Fabricating the provisional restorations on a cast of the actual tooth preparations has the following advantages compared to making initial temporary restorations from a wax-up alone:

- Additional space for acrylic resin allows for creation of ideal aesthetic, occlusal and functional form

- Additional bulk of material and extra-oral processing provides increased durability, strength, biocompatibility and aesthetics

- Marginal accuracy of fit and the creation of the correct emergence profile allows for good soft tissue response and biologic seal

- A three-dimensional assessment of tooth reduction/preparation design can be made in the laboratory. If necessary, modifications are then made to the tooth preparation.

The definitive gold onlays were cemented with zinc phosphate (ZnP) cement under rubber dam (Figs 8-9). Although zinc phosphate may be considered by some to be obsolete compared to newer resin based cements, it is still the cement of choice under precision gold onlays that have been prepared with correct macromechanical resistance features. The reasons for this are:

- Resin cements have increased film thickness and prevent full and precise seating of the fine metal margins

- The extended setting time $\mathrm{ZnP}$ cement allows for precise finishing and burnishing of the gold margins prior to the cement setting. This hermetic seal eliminates any potential for future washout of cement. Burnishing of margins is not possible with a set resin cement

- Resin cements have limited long term clinical research and lack a long term clinical track record. $\mathrm{ZnP}$ has proven itself clinically to survive for extremely long time periods under well sealed and precisely fitting gold onlays.

It has also been shown that resistance form is the most important aspect for long term retention of restorations. ${ }^{12}$ Resin cements should only be used if adequate resistance form cannot be achieved.

The provisional restorations should be well fitting and have the correct emergence profile to condition the gingival margin tissues which in turn facilitates making of the final impression without concern for inflamed or overgrown gingival tissues (Figs 10-12). ${ }^{13,14}$ If any gingival recession is observed in this stage, the associated margins are reprepared and the overlying provisional crown remarginated. A precise occlusal relationship is incorporated into the provisional restorations and once in the mouth, the occlusal scheme is verified and minor adjustments made (Fig. 13). The occlusal harmony/function is observed over a period of time and adjusted to ensure comfort and stability.

Once the dentist and patient are satisfied with the biomechanical integrity, occlusal stability and function, and aesthetic appearance, the final impression is made.

\section{Stage 4 - definitive impressions}

Complete arch impressions of multiple tooth preparations are fraught with difficulties and potential complications such as:

- Distortion of material due to undercuts created by divergent teeth

- Insufficient working time

- Inability to control moisture

- Increased stress for the dentist and patient

- Increased patient discomfort

- Difficulty in atraumatic gingival retraction around multiple teeth and adjacent anterior teeth

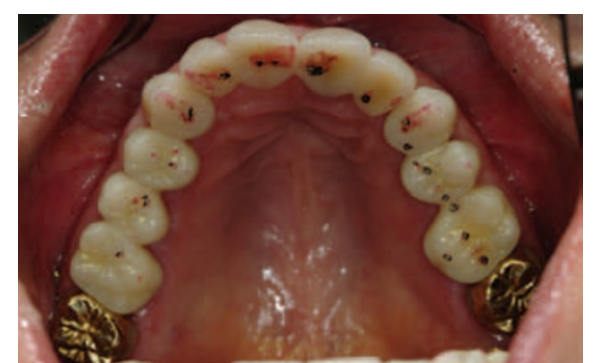

Fig. 13 Refinement of occlusal scheme on definitive gold and laboratory processed, provisional restorations

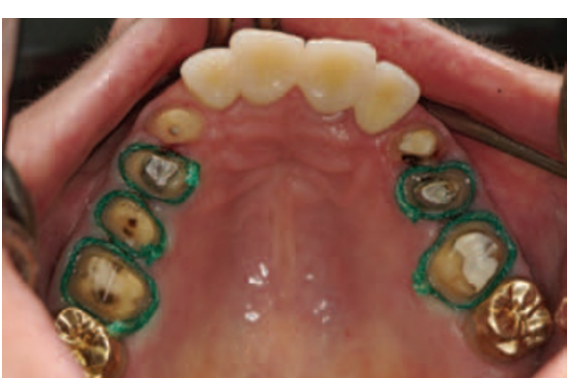

Fig. 14 Posterior teeth with retraction cord ready for final sectional impression

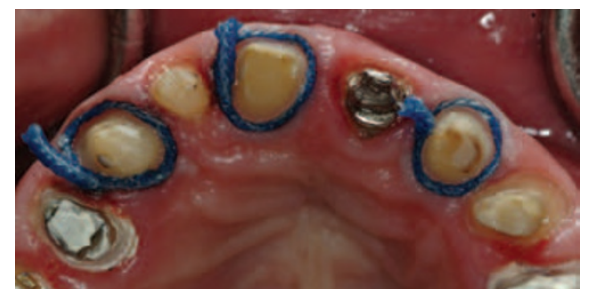

Fig. 15 Alternate anterior teeth with retraction cord ready for one of the final impressions of three anterior teeth

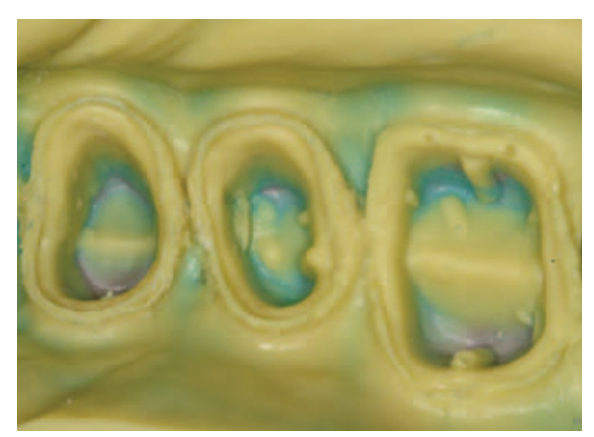

Fig. 16 Sectional impression of posterior teeth showing capture of all margins in a controlled and stress free manner

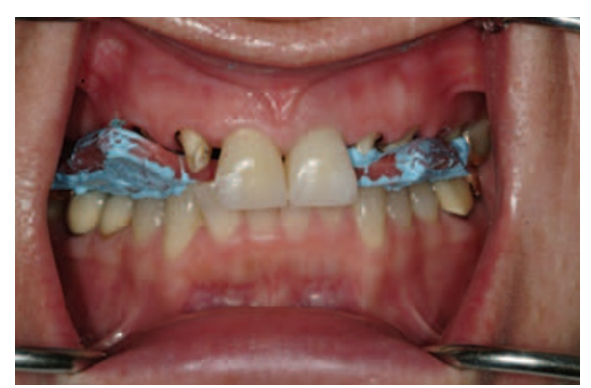

Fig. 17 Bite registration record utilising anterior provisional restorations as a vertical stop at correct VDO 


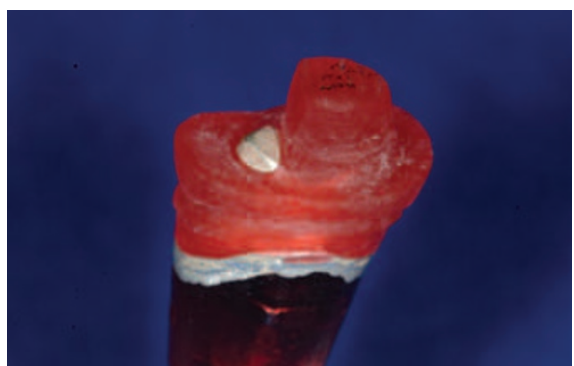

Fig. 18 Design of resin copings. Note window for assessing correct intra-oral fit and precise marginal fit to allow intra-oral verification of die accuracy

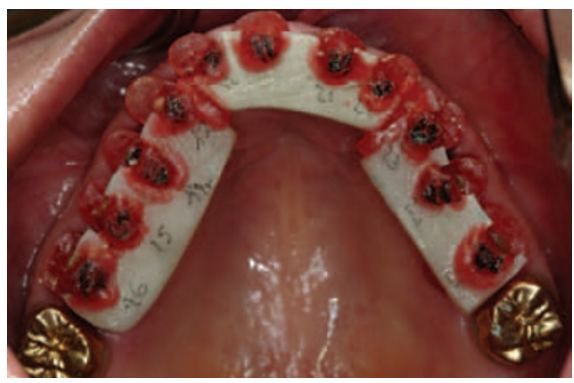

Fig. 19 Resin copings in place and luted together ready for pickup impression in a custom impression tray

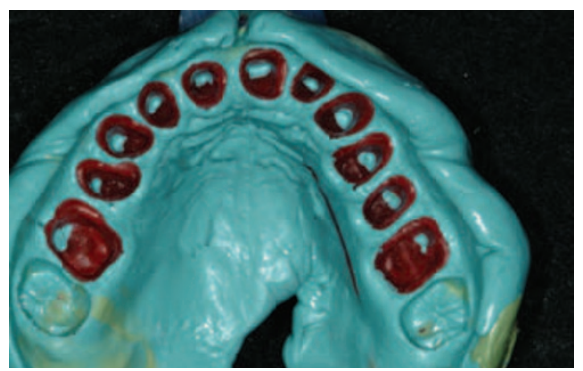

Fig. 20 Pickup impression in custom tray using polyvinylsiloxane impression material

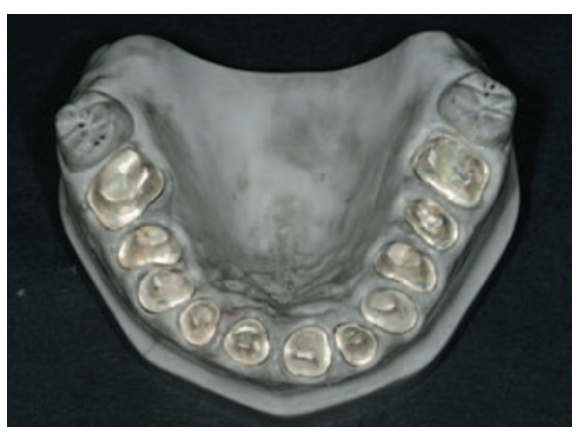

Fig. 21 Master model with original removable silver dies. Note impression of the soft tissues in a non retracted state

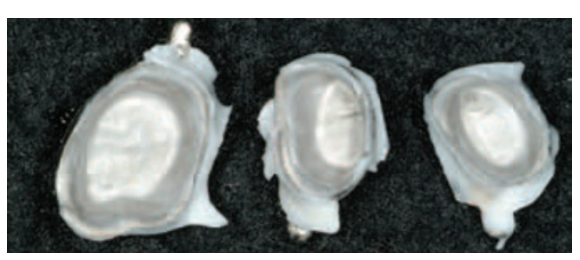

Fig. 22 Fit checker inside metal copings. Note thin internal uniform layer and precise marginal adaptation

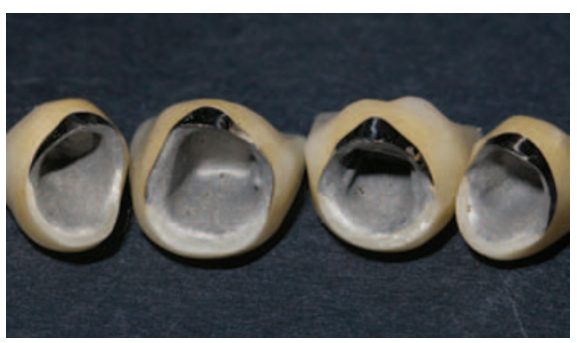

Fig. 23 Final anterior restorations. Note clean internal surface of castings and transition from porcelain shoulder margin in visible areas to metal chamfer margins in non-visible areas

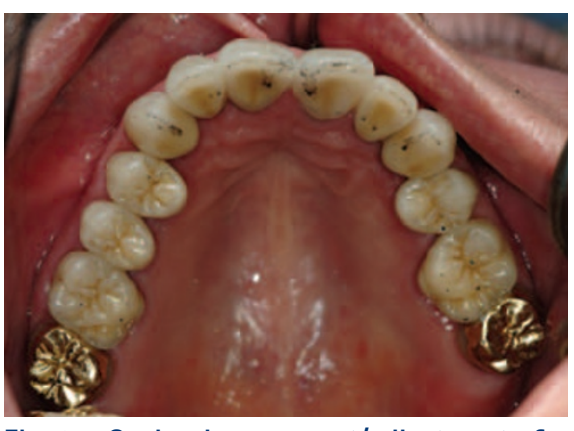

Fig. 24 Occlusal assessment/adjustment of final restorations

- Excessive trauma to soft tissues by excessive and overaggressive retraction to compensate for additional working time required to obtain a full arch impression.

For these reasons, it is not possible to predictable and precisely capture all margins in a full arch impression. The success of an impression is relative to the understanding of the person examining it and the intensity and magnification at which it is assessed. A brief glance or a slide in a lecture is no indication of success.

The technique utilised in this case involved making multiple sectional impressions and then making a pickup of resin copings made to fit on each tooth preparation. It was originally described to be used with copper band impressions and has undergone various modifications/adaptations to accommodate modern materials. ${ }^{15-17}$ It eliminates the use of two different dies for each restoration and also eliminates the need for attempting to impress adjacent anterior teeth with thin interproximal tissue that could be damaged during the gingival retraction.

The following sectional impressions were made:
- 6 posterior teeth $(16,15,14$ and 26, 25, 24) (Fig. 14)

- 3 alternate anterior teeth $(13,11,22)$

- 3 alternate anterior teeth $(12,21,23)$ (Fig. 15)

It is far easier and predictable to obtain clinical precision with smaller sectional impressions rather than with a single full arch impression (Fig. 16). A bite registration record was made using brittle wax and bite registration paste utilising the two anterior provisional restorations as a stop to record the desired VDO (Fig. 17).

In the laboratory, individual silver plated dies were then produced from the sectional impressions ${ }^{18}$ and resin copings were made on each die. A small window was made on the labio-occlusal surface of each coping to allow intra-oral verification that each coping is fully seated (Fig. 18). These copings need to be very precise with a perfect fit to prevent impression material getting under them in the pickup impression and to allow the accuracy of the dies to be verified in the mouth.

In the mouth, the copings were relocated precisely on their specific tooth preparations and their fit was assessed. If a coping does not fully seat on the preparation, yet it fits the die, a new impression and resin coping must be made for that individual tooth before a pickup impression can be made. The copings were then luted, with a minimal amount of acrylic pattern resin, to a custom made key for increased stability and rigidity (Fig. 19). If the tooth preparations are very divergent, the copings may be luted together in smaller sections. A pickup impression utilising polyvinylsiloxane impression material in a custom tray was then made (Fig. 20).

In the laboratory, the original silver dies were precisely relocated into their copings and a master model was made (Fig. 21). This model unites all the individual dies and allows the individual dies to be removed for fabrication of the restorations. An additional use of the pickup impression is to capture the soft tissues in their non-retracted healthy state. Conventional final impressions capture the tissues in a retracted and displaced state. This gives a cast with 


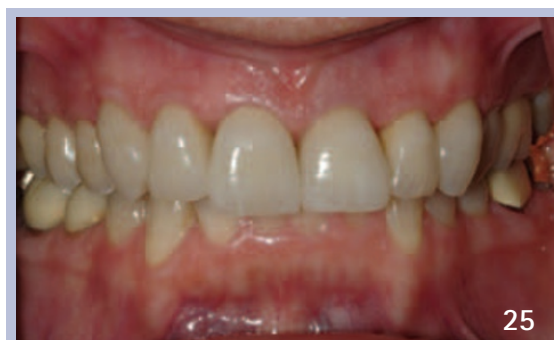

25
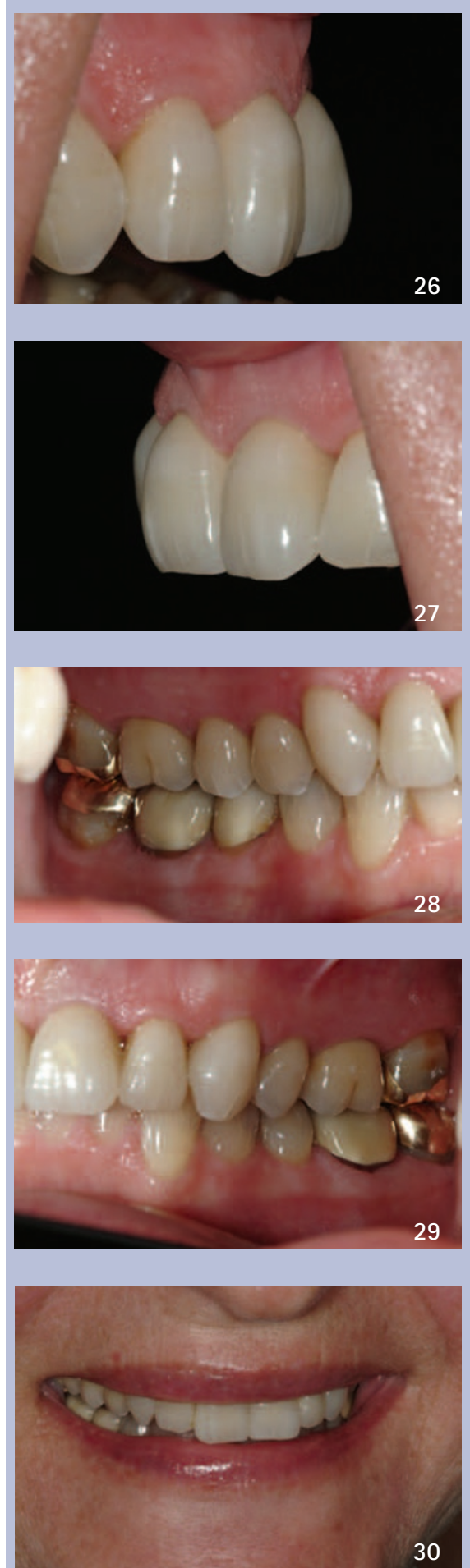

Figs 25-30 Final restorations in place. Note detail to emergence profile and natural morphology. Despite having metal cores, the margins of the crowns do not detract from the aesthetic appearance distorted soft tissues that does not realistically replicate the resting state of the interdental papillae making it difficult for the technician to know where to place the contact point. ${ }^{19}$

The disadvantages of this pickup technique include:

- The need for the use of metal dies, as making resin copings on traditional gypsum dies tends to damage the dies

- Meticulous attention to precision is needed during coping fabrication and during the coping pickup and the die relocation stages. Any inaccuracies in these stages will lead to an error in the relationship between the individual dies in the master model. This in turn would lead to excessive adjustments to the contact points and the occlusion at the final cementation stage.

Once the master model had been constructed, the underlying metal copings were fabricated according to a strict scientific and technical protocol as described by Sgro. ${ }^{20}$

\section{Stage 5 - Fitting and cementation of final restorations}

Once the metal copings had been fabricated, the internal fit was checked intraorally, using a silicone based fit checking material (Fit Checker, GC, Tokyo, Japan) (Fig. 22). Studies have shown that this is an important step and significantly improves seating of the restorations. ${ }^{21,22}$ At this stage the accuracy of the bite relationship record was also verified. If necessary, a remount procedure as described by Eggleston ${ }^{23}$ is carried out before proceeding with the porcelain application.

The porcelain application was then carried out and completed to a bisque bake stage. The restorations in the bisque bake stage were tried in the mouth and the aesthetics, contact points and occlusion were assessed. Minor changes were noted and carried out in the laboratory prior to completing the porcelain firing and final glazing (Fig. 23).

The final restorations were then cemented. The cement used was a resin modified glass ionomer (Fuji Plus, GC, Tokyo, Japan). It is important to have adequate moisture control during cemenation and it is advisable to place fine retraction cords around teeth with intrasulcular margins prior to cementation. These retraction cords serve three purposes:

- Prevent sulcular fluid from contaminating the cement during setting

- Ensure fully exposed margins without any soft tissue impingement during cementation.

- Prevent excess cement from going too far subgingival.

As mentioned earlier, with adequate resistance form in a tooth preparation, it is not necessary to rely on retention with resin cements. Resin cements are extremely sensitive to moisture and the same precaution should be used with resin cements as with resin restorations ie adequate moisture control with rubber dam. This is usually not possible with full crown preparations that have intracrevicular margins in some visible areas.

Following adequate setting of the cement, all the excess cement was removed from the margins. This is a time consuming procedure and must be done meticulously with a sickle scaler or similar instrument. The dental assistant should spray a constant stream of air between the gingival margin and the crown. This has the effect of forcing the gingival away from the restoration allowing direct visualisation of the margin and any cement residue. Only once all the excess cement has been removed, is the retraction cord removed.

Following cementation, the final occlusal assessment and adjustment was carried out (Fig. 24). This is an essential step as there are always minor occlusal discrepancies following cementation and it has been shown that control of the forces on restorations is a key factor in their long term prognosis. ${ }^{24}$ If attention to detail has been followed, and accuracy verified at all staged, the postcementation occlusal adjustment should be minimal (Figs 25-30). The adage that 'the mouth is the best articulator' should only be applied at this very final stage of intra-oral occlusal adjustment. If the dental articulator and bite registration records have not been precisely used during fabrication of the restorations, the dentist will be left with large occlusal discrepancies which will need to be adjusted in the mouth. Besides taking 
time and destroying the confidence of the patient, the morphology of the final restorations will be mutilated.

\section{DISCUSSION}

In complex full arch restoration cases, it is essential to maintain control at all times and ensure precision at all stages. Treatment should be carried out in stages ensuring the success of each stage before proceeding on to the next stage. In complex cases there is also the potential for small errors to be magnified as treatment stages and therefore it is essential to ensure accuracy and precision at each stage and also verify and make adjustments along the way to minimise inaccuracies at the end of treatment.

The author would like to acknowledge Mr Salvatore Sgro of L'Eccellenza Odontotecnica S.r.l, Rome, Italy for the excellent technical work and inspiration.

1. Koka S, Linebaugh M L. A technique to reposition dies on the working model during fixed prosthodontic procedures. J Prosthet Dent 1993 69: 537-538.

2. Hochstedler J L, Elliott R B. A method to position replacement dies accurately on a fixed partial denture master working cast. J Prosthet Dent 1995; 74: 198-201.
3. Morgano S M, Milot P, Ducharme P, Lynda Rose L. Ability of various impression materials to produce duplicate dies from successive impressions. $J$ Prosthet Dent 1995: 73: 333-340.

4. Gracis S. Clinical considerations and rationale for the use of simplified instrumentation in occlusal rehabilitation. Part 1: Mounting of the models on the articulator. Int J Periodontics Restorative Dent 2003; 23: 57-67.

5. Gracis S. Clinical considerations and rationale for the use of simplified instrumentation in occlusal rehabilitation. Part 2: setting of the articulator and occlusal optimization. Int J Periodontics Restorative Dent 2003: 23: 139-145.

6. Parker M H. Resistance form in tooth preparation. Dent Clin North Am 2004; 48: 387-396.

7. Burns D R, Beck D A, Nelson S K Committee on Research in Fixed Prosthodontics of the Academy of Fixed Prosthodontics. A review of selected dental literature on contemporary provisional fixed prosthodontic treatment: report of the Committee on Research in Fixed Prosthodontics of the Academy of Fixed Prosthodontics. J Prosthet Dent 2003; 5: 474-497.

8. Wang R L, Moore B K, Goodacre C J, Swartz M L, Andres C J. A comparison of resins for fabricating provisional fixed restorations. Int J Prosthodont 1989: 2: 173-184.

9. Dawson P. Smile design. In Dawson P. Functional occlusion. From TMJ to smile design. pp155-156. Mosby, 2007.

10. Crispin B J, Watson J F, Caputo A A. The marginal accuracy of treatment restorations: a comparative analysis. J Prosthet Dent 1980; 3: 283-290.

11. Sgrò S, Mizrahi B. Individualizing esthetic treatment outcomes: planning and fabrication. Quintessence Dent Technol 2007: 155-175.

12. Trier A C, Parker M H, Cameron S M, Brousseau J S. Evaluation of resistance form of dislodged crowns and retainers. J Prosthet Dent 1998; 80: 405-409.

13. Shavell H M. The periodontal-restorative interface in fixed prosthodontics: tooth preparation, provisionalization, and biologic final impressions. Part Pract Periodontics Aesthet Dent 1994; 6: 33-44.

14. Shavell H M. The periodontal-restorative interface in fixed prosthodontics: tooth preparation, provisionalization, and biologic final impressions. Part II. Pract Periodontics Aesthet Dent 1994. 6: 49-60.

15. Zuckerman $\mathrm{G}$ R. Dies with resin copings for accurate registrations. J Prosthet Dent 1992; 67: 37-40.

16. Crispin B. Acrylic resin copings: an adjunct to fixed restorative dentistry. J Prosthet Dent 1978; 39: 632-636.

17. Messing J J. Copper band technique. Br Dent J 1965: 119: 246-248.

18. Sgrò S, Eliseo M. Galvanoplastica in argento. Ottimizazione del metodo. Dental Labor 1998; 5: 444-456.

19. Touati B, Etienne J M. Improved shape and emergence profile in an extensive ceramic rehabilitation. Pract Periodontics Aesthet Dent 1998; 10: 129-135.

20. Sgrò S. Principles of the metal framework design in metal-ceramic reconstructions. Quintessence Dent Technol 2002; 25: 21-52.

21. White S N, Sorensen J A, Kang S K. Improved marginal seating of cast restorations using a silicone disclosing medium. Int J Prosthodont 1991. 4: 323-326.

22. Ushiwata O, de Moraes J V, Bottino M A, da Silva E G. Marginal fit of nickel-chromium copings before and after internal adjustments with duplicated stone dies and disclosing agent. J Prosthet Dent 2000; 83: 634-643.

23. Eggleston D W. Advantages and use of the remount for fixed prosthodontics. J Prosthet Dent 1980; 43: 627-633.

24. Torbjörner A, LD S, Fransson B. Biomechanical aspects of prosthetic treatment of structurally compromised teeth. Int J Prosthodont 2004; 17: 135-141. 